\title{
Assessing balance through the use of a low-cost head-mounted display in older adults: a pilot study
}

This article was published in the following Dove Press journal:

Clinical Interventions in Aging

26 August 2017

Number of times this article has been viewed

\author{
Santiago J Saldana' \\ Anthony P Marsh ${ }^{2}$ \\ W Jack Rejeski \\ Jack K Haberl ${ }^{2}$ \\ Peggy $\mathrm{Wu}^{3}$ \\ Scott Rosenthal ${ }^{4}$ \\ Edward H Ip'
}

'Department of Biostatistical Sciences, Wake Forest School of Medicine, ${ }^{2}$ Department of Health and Exercise Science, Wake Forest University, Winston-Salem, NC, ${ }^{3}$ Research and Development, Smart Information Flow Technologies, Minneapolis, MN, ${ }^{4} \mathrm{Wake}$ Forest School of Medicine, WinstonSalem, NC, USA
Correspondence: Santiago J Saldana Department of Biostatistical Sciences, Wake Forest School of Medicine, Medical Center Boulevard, Winston-Salem, NC 27I57, USA

Email ssaldana@wakehealth.edu
Introduction: As the population ages, the prevention of falls is an increasingly important public health problem. Balance assessment forms an important component of fall-prevention programs for older adults. The recent development of cost-effective and highly responsive virtual reality (VR) systems means new methods of balance assessment are feasible in a clinical setting. This proof-of-concept study made use of the submillimeter tracking built into modern VR head-mounted displays (VRHMDs) to assess balance through the use of visual-vestibular conflict. The objective of this study was to evaluate the validity, acceptability, and reliability of using a VRHMD to assess balance in older adults.

Materials and methods: Validity was assessed by comparing measurements from the VRHMD to measurements of postural sway from a force plate. Acceptability was assessed through the use of the Simulator Sickness Questionnaire pre- and postexposure to assess possible side effects of the visual-vestibular conflict. Reliability was assessed by measuring correlations between repeated measurements 1 week apart. Variables of possible importance that were found to be reliable $(r \geq 0.9)$ between tests separated by a week were then tested for differences compared to a control group. Assessment was performed as a cross-sectional single-site community center-based study in 13 older adults ( $\geq 65$ years old, $80.2 \pm 7.3$ years old, $77 \%$ female, five at risk of falls, eight controls). The VR balance assessment consisted of four modules: a baseline module, a reaction module, a balance module, and a seated assessment.

Results: There was a significant difference in the rate at which participants with a risk of falls changed their tilt in the anteroposterior direction compared to the control group. Participants with a risk of falls changed their tilt in the anteroposterior direction at $0.7 \%$ second vs $0.4 \%$ second for those without a history of falls. No significant differences were found between pre/ postassessment for oculomotor score or total Simulator Sickness Questionnaire score. Both the force plate and the head-mounted display balance-assessment system were able to detect differences between conditions meant to mask visual and proprioceptive information.

Conclusion: This VRHMD is both affordable and portable, causes minimal simulator sickness, and produces repeatable results that can be used to assess balance in older adults.

Keywords: virtual reality, balance, falls, elderly

\section{Introduction}

Around the world, populations are aging at an accelerated rate, with the number of elderly due to double by $2050 .{ }^{1}$ In older populations, injuries and costs due to falls are disproportionally high compared to younger populations, and their prevention is an important public health problem. ${ }^{2,3}$ Clinical practice guidelines recommend a multifactorial approach to prevent falls, including asking about risky activities, reviewing medications, and assessing gait and balance. ${ }^{4,5}$ 
There are various methods to assess fall risk, such as surveys, physical tests, and perturbation-based assessments, each having strengths and limitations. Surveys are useful in the assessment of external risk factors for falls, but must be combined with physical measurements to assess intrinsic risk factors. Physical assessments, such as the timed "up and go", gait speed, Short Physical Performance battery, and the Tinetti balance- and gait-evaluation scale are easy to administer, but lack ecological validity, as well as the ability to isolate specific sensory defects. ${ }^{6}$ Such tests as one-legged balance are useful for information gathering, but not diagnosis, as standards for diagnosis have not been set. ${ }^{7}$ Perturbation-based assessments allow the ability to test individual sensory systems; however, the equipment needed can be expensive and typically lacks portability. ${ }^{8}$

Maintenance of balance is a complex process involving the processing of information from the visual, somatosensory, and vestibular systems. ${ }^{9}$ Current state-of-the-art equipment, such as the EquiTest (Neurocom, Clackamas, OR, USA), measures the relative contribution of each system to maintain balance using a structured protocol. ${ }^{10}$ The EquiTest has a tilting platform to mask information to the somatosensory system and a tilting facade to confound information from the visual system, increasing cost and reducing portability. In contrast, the Balance Error Scoring System (BESS) uses a foam balance pad to reduce somatosensory feedback and has the user close their eyes to eliminate visual feedback. ${ }^{11}$ While the balance pad provides an affordable alternative to mechanical perturbation, a more advanced technique is needed for the visual system, particularly since it has been suggested that older adults may attend to visual information more closely, due to reduced vestibular and postural sensation. ${ }^{12}$

The recent availability of low-cost virtual reality (VR) systems designed for the retail market provides a new medium to immerse a user's visual field completely while simultaneously adding a sense of depth to a user's experience. These systems have been quickly adopted, and current work shows that the addition of VR can provide a positive impact on intervention programs targeting adults at risk of falls, where the addition of a VR component to a treadmill training program reduced fall rates compared to treadmill training alone. ${ }^{13}$ In the field of balance assessment, recent work has shown that it is also possible to produce results comparable to an EquiTest assessment using VR in combination with an inexpensive force plate to replicate the abilities of the EquiTest. ${ }^{14}$ Additionally, traditional posturography has shown significant differences in static balance between fallers and nonfallers in several studies, including velocity, displacement, and area. ${ }^{15}$ Preliminary work also showed that the use of VR headsets for balance assessment is safe, with the risk for destabilization being similar to standing with eyes closed. ${ }^{16}$ While work has been done on assessing balance using commercially available modern VR head-mounted displays (VRHMDs), , ${ }^{14,16,17}$ as far as we know, none of the current work has targeted older adults who are at risk of falls. The objective of this proof-of-concept study was to provide preliminary evidence for the validity, reliability, and acceptability of using a VRHMD to assess balance in older adults.

Validity was assessed by comparing measurements from the VRHMD to measurements of postural sway from a force plate. Acceptability was assessed through the use of preand postexposure Simulator Sickness Questionnaire (SSQ) scores to assess possible side effects of the visual-vestibular conflict used during assessment. Reliability was assessed by measuring correlations between repeated measurements 1 week apart.

\section{Materials and methods Subjects}

Participants were recruited from a continuing-care retirement community. They were asked a series of questions via a phone screener to make sure they qualified for the study. The questions included the STRIDE study-inclusion criteria for fall risk, specifically "Have you fallen and hurt yourself in the past year?", "Have you fallen two or more times in the past year?", and "Are you afraid that you might fall because of balance or walking problems?". ${ }^{18}$ An answer of yes to any of these questions classifies a participant as being higher risk for falls. Participants were only excluded if the required number (maximum ten) of high-risk and low-risk participants had already been recruited. Based on this information, participants were classified into two groups (eight at risk of falls, five controls). The STRIDE protocol was used, as it was a large multicenter study aimed at evaluating the effectiveness of strategies to reduce serious fall-related injuries in older adults. There were no group differences in age, body mass, or height (Table 1). Inclusion criteria were $\geq 65$ years old, no history of major cardiovascular events, such as stroke, no cognitive impairment, able to stand and ambulate independently, and comfortable wearing a VRHMD in a static environment. These requirements necessitated the use of a six-item phone screener ${ }^{19}$ and an initial in-person assessment. The phone screener is a widely used instrument for the identification of subjects with cognitive impairment. During the initial in-person assessment, participants completed the Montreal Cognitive Assessment (MoCA) cognitive test ${ }^{20}$ and were required to score $\geq 21$ for inclusion in the study. Participants also completed the Mobile Assessment Test - short form ${ }^{21}$ 
Table I Subject characteristics (mean \pm SD)

\begin{tabular}{llll}
\hline Variable & $\begin{array}{l}\text { At risk of } \\
\text { falls }(\mathbf{n}=5)\end{array}$ & $\begin{array}{l}\text { Low risk of } \\
\text { falls }(\mathbf{n}=\mathbf{8})\end{array}$ & P-value \\
\hline Sex (female), n (\%) & $3(60 \%)$ & $7(87.5 \%)$ & 0.25 \\
Age (years) & $78.4 \pm 9.37$ & $81.4 \pm 6.25$ & 0.5 \\
Height (m) & $0.6 \pm 0.04$ & $1.4 \pm 0.53$ & 0.6 \\
Mass (kg) & $67.5 \pm 8.05$ & $70.4 \pm 10.18$ & 0.42 \\
MoCA & $28.2 \pm 2.17$ & $27.1 \pm 2.03$ & 0.38 \\
MAT-SF (range 30-70) & $50 \pm 9.66$ & $59.9 \pm 7.66$ & 0.06 \\
One-leg stance (seconds) & $10.5 \pm 16.63$ & $8.5 \pm 5.02$ & 0.76 \\
\hline
\end{tabular}

Abbreviations: MoCA, Montreal Cognitive Assessment; MAT-SF, Mobility Assessment Tool - short form.

(MAT-SF) tablet-based mobility-assessment tool and a score in the tenth percentile or higher was required for inclusion. This was included for participant safety, as basic ambulation was necessary for assessment. The Wake Forest School of Medicine Institutional Review Board approved the study design and informed-consent document, including video taping consent, and all participants provided informed consent prior to any data collection and were compensated for their time.

\section{Design and procedures}

The study was designed to assess the validity, acceptability, and reliability of using a VRHMD to assess sway. Validity was assessed by evaluating differences between older adults at risk of falls and a control group, as well as comparing measurements from the VRHMD to measurements of postural sway from a force plate. Acceptability was evaluated by the $\mathrm{SSQ}^{22}$ to assess possible side effects, such as motion sickness, which could occur due to the visual-vestibular conflict used during assessment. ${ }^{23}$ Reliability was assessed by using repeated measurements 1 week apart.

The study consisted of two visits 1 week apart, which involved demographic, cognitive, and VR measurements. During the first visit, the MoCA and MAT-SF were assessed as part of the screening process. ${ }^{20,21}$ Height and body mass were measured using a calibrated stadiometer and scale. Participants were provided a short familiarization period wearing the VRHMD in a static desk scene. After familiarization, participants completed a one-leg stance test ${ }^{7}$ with eyes open, to be used as a simple comparison of balance ability between those with and without a risk of falls. Participants were then administered a preexposure $\mathrm{SSQ}^{22}$ to measure possible existing simulator sickness symptoms.

During testing, participants were protected against possible falls via a "safety enclosure" consisting of a Clinton balance platform. ${ }^{24}$ The platform was modified with cross braces and 1-inch $(2.54 \mathrm{~cm})$ foam tubing (Figure 1). A test administrator demonstrated the two positions used during testing: feet together and feet in a semitandem (modified

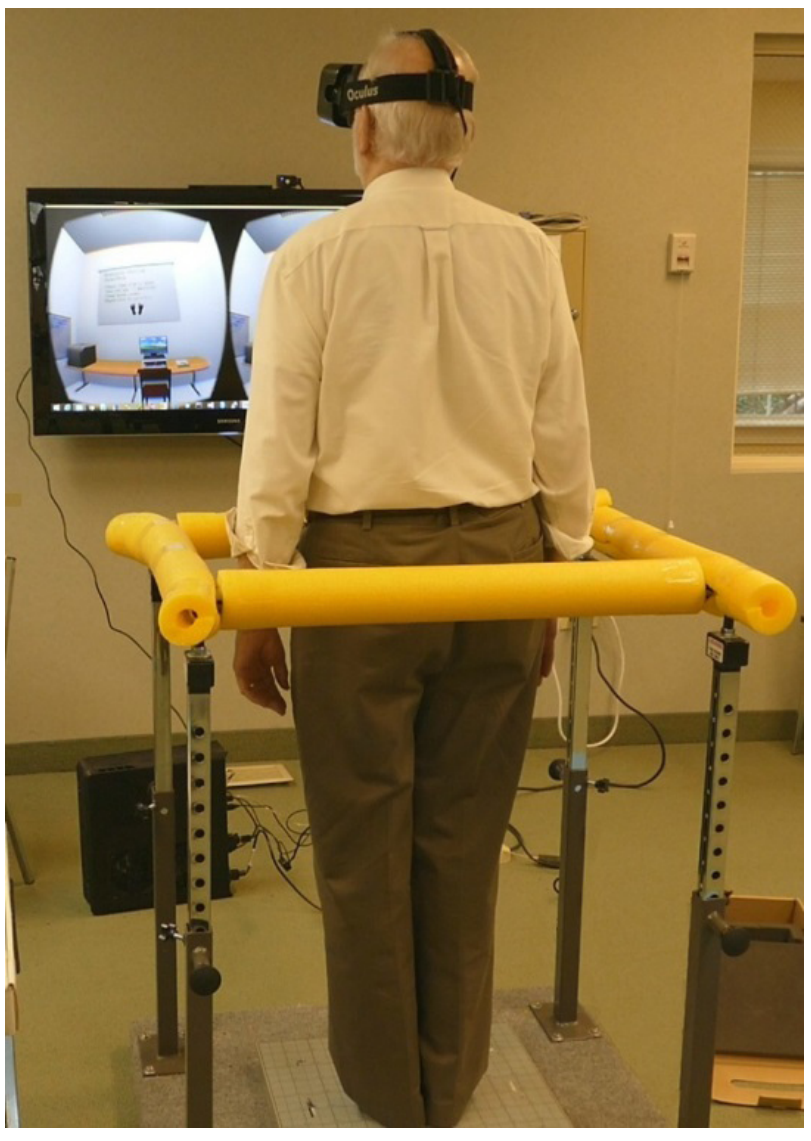

Figure I On-site testing environment.

Notes: The participant is shown standing with feet together prior to a baseline balance assessment. The participant is standing on the force plate and surrounded by the Clinton balance platform to prevent a fall to the ground in the event of a loss of balance. The virtual environment as seen in the VRHMD presents instructions for each module to the participant, and the participant is instructed to read them aloud. The monitor on the wall allows the staff member to see what the participant is seeing at each stage of the balance protocol. Measurements from the VRHMD and the force plate were synchronized and recorded simultaneously.

Abbreviation: VRHMD, virtual-reality head-mounted display.

Romberg) stance. These stances correspond to those used in the BESS and previous work. ${ }^{11,25}$ For the seated portion, a chair was placed directly on the force plate with chair legs equidistant from the corners of the force plate. Measurements from the force plate were calibrated, so that the chair did not have an effect on the readings. The participant was assisted onto the AMTI AccuSway force plate (Advanced Mechanical Technology, Watertown, MA, USA) contained within the safety enclosure for administration of the VR portion of the assessment.

\section{VR modules}

The VR assessment included four modules: baseline, reaction, balance, and seated. Each module presented a virtualclassroom environment to the participant with instructions presented on a virtual screen directly ahead of the participant's view (Figure 1). 


\section{Baseline}

To determine baseline postural sway, the participant was asked to stand with feet side by side for 10 seconds with eyes open and then with eyes closed. The participant was then asked to repeat this procedure with feet in a semitandem stance with foot positioning identical to that used in the BESS. $^{11}$

\section{Balance}

Participants repeated the baseline module with feet in a semitandem stance and with feet side by side. The balance module required the participant to stand as still as possible while the room view alternated between rotating $30^{\circ}$ from center in the anteroposterior and then the mediolateral directions (Figure 1). The period of rotation was 10 seconds for a complete oscillation between $-30^{\circ}$ to $30^{\circ}$ and back to $-30^{\circ}$. This was repeated four times, with a break in between.

\section{Reaction}

In the reaction module, a participant's view pitched forward and back between one and five times. The participant was asked to match his/her sway to that of the room, so that the room appeared not to move. As the room view passed the neutral position, it would tilt left/right once during each trial, and the participant was asked to move in that direction.

\section{Seated}

The seated module followed the same protocol as the baseline and balance modules with the use of a chair placed centrally on the force plate. The purpose of the seated module was to see if any differences were discernible from this position, as it would eliminate the need for a safety enclosure.

Each condition for the baseline and balance modules was repeated four times. Participants also repeated the steps outlined in the baseline and balance modules with the use of a balance pad (Airex AG, Sins, Switzerland). This increased difficulty which reduced the chance of ceiling effects and reduced somatosensory feedback for isolating balance performance and available sensory-system information.

\section{Equipment}

Force-plate measurements were acquired at $100 \mathrm{~Hz}$ and synchronized with Rift DK2 (Oculus VR, Irvine, CA, USA) VRHMD measurements of $75 \mathrm{~Hz}$. The DK2 uses a combination of inertial sensors and an external synchronized camera to provide accurate $6^{\circ}$-of-freedom tracking of spatial position with millimeter accuracy. It has been designed with a low-persistence panel, which greatly reduces motion blur visible on the screen. Motion blur has been shown to contribute toward increased simulator sickness. ${ }^{26}$ The DK2 software-development kit provided position and rotation of the headset. The kit also provided an estimated eye height and depth offset from the point of articulation (neck). Using this information and the current orientation of the headset, it was possible to subtract the offset caused by rotating the headset during assessment.

\section{Measurements}

Velocity, amplitude, maximum displacement, average displacement, and area were calculated for both force-plate and VRHMD measurements for both anteroposterior and mediolateral directions with respect to the center of pressure/ center of tilt, respectively. These variables were chosen as they represent some of the most common variables used for traditional global analysis of center of pressure, which is used in traditional posturgraphy. ${ }^{27}$ While specific findings vary by study, it is generally considered that those with worse balance have greater postural sway and are at greater risk of falls. ${ }^{28}$ Velocity measured the change in position, amplitude measured the maximum range of measurements, displacement measured the maximum position, and averaged displacement measured the average position over the trial. Raw measurements for both the force plate and the VRHMD were in millimeters, and force-plate measurements corresponded directly to the center of pressure, whereas the VRHMD measurements provided the 3-D position of the VRHMD relative to the measurement camera. For measurements taken from the VRHMD, tilt in degrees was calculated using knowledge of the participant's height, and the VRHMD orientation adjusted head position. Head positions and center positions were measured relative to an initial position, which was reset between each trial. Measurements of the center of pressure were obtained from the force plate. Area was also calculated as a $95 \% \mathrm{CI}$ ellipse, as outlined by Duarte and Freitas. ${ }^{27}$

\section{Statistical analysis}

Data preprocessing included calculations for velocity, amplitude, maximum displacement, average displacement, and area, and values were calculated using R 3.2.1. Statistical analysis included descriptive statistics, paired $t$-tests, ShapiroWilk normality tests, pooled and Satterthwaite tests were completed using SAS 9.4 (SAS Institute, Cary, NC, USA). Shapiro-Wilk normality tests were used to determine whether to use the pooled (equal variances) or Satterthwaite (unequal variances) results for the baseline-group comparisons. 
Normality results were also used in the SSQ-score pre- and postexposure comparisons, where a paired $t$-test was used if distributions were normal and a signed-rank test used otherwise. Test-retest measurement reliability was assessed using Pearson's correlation coefficient and comparing measurements for the whole cohort.

\section{Results}

There were no significant between-group differences on demographic characteristics, MoCA, MAT-SF, or one-leg stance (Table 1). Only during visit 1 was there any significant difference in any component of the SSQ, where there was a significant difference in only the nausea subscale. As a whole, participants had an average preexposure SSQ score of $1.62 \pm 2.66$ and a postexposure score of $3 \pm 3.54$ for visit 1 and $2.33 \pm 4.5$ and $2.58 \pm 3.63$ for visit 2 . There was a preexposure-postexposure average difference of $-1.31 \pm 1.8$, where participants had lower scores before exposure to the VRHMD (Table 2). The maximum possible score for the nausea subscale is 27 . However, one participant did not complete their second visit, reporting experiencing simulator sickness after the first visit.

Paired $t$-tests comparing anteroposterior amplitude for VRHMD and force-plate measurements showed that both techniques were capable of finding significant differences among all but one of five conditions, (eyes closed, eyes open/room moving, eyes open/balance pad, eyes closed/ balance pad, and eyes open/balance pad/room moving) from a baseline condition of eyes open/feet together/no foam (Table 3), which is the same approach used when comparing accelerometer-tilt and force-plate measurements, as well as the same comparison used during the EquiTest. ${ }^{10,29}$ Based on data from both controls and those with a history of falls, neither the VRHMD nor the force plate was capable of distinguishing between eyes open or closed under baseline conditions. In order to reduce type I error in finding differences between the control group and those with a history

Table 2 Pre/post-Simulator Sickness Questionnaire score differences

\begin{tabular}{lllll}
\hline & Visit & Mean & SD & P-value* \\
\hline Nausea subscale difference & $\mathrm{I}$ & $-\mathrm{I} .3 \mathrm{I}$ & $\mathrm{I} .8$ & 0.02 \\
Oculomotor subscale difference & $\mathrm{I}$ & -0.08 & $\mathrm{I} .04$ & 0.98 \\
Total score difference & $\mathrm{I}$ & $-\mathrm{I} .38$ & 2.29 & 0.05 \\
Nausea subscale difference & 2 & 0.08 & $\mathrm{I} .83$ & 0.88 \\
Oculomotor subscale difference & 2 & -0.33 & 0.89 & 0.36 \\
Total score difference & 2 & -0.25 & $\mathrm{I.91}$ & 0.63
\end{tabular}

Notes: $* P$-value from a one-sample $t$-test if Shapiro-Wilk test $\geq 0.05$, signed-rank test otherwise. Maximum scores: nausea 27, oculomotor 2I, total 48. Abbreviation: SD, standard deviation.
Table 3 Paired $t$-test $P$-value comparisons with baseline eyesopen condition

\begin{tabular}{|c|c|c|c|c|}
\hline & \multirow{2}{*}{\multicolumn{2}{|c|}{$\begin{array}{l}\text { Plate, } \\
\text { anteroposterior } \\
\text { amplitude } \\
\text { Visit }\end{array}$}} & \multirow{2}{*}{\multicolumn{2}{|c|}{$\begin{array}{l}\text { VRHMD, } \\
\text { anteroposterior } \\
\text { amplitude } \\
\text { Visit }\end{array}$}} \\
\hline & & & & \\
\hline & I & 2 & I & 2 \\
\hline Eyes closed & 0.272 & 0.9123 & 0.8988 & 0.4085 \\
\hline Eyes open, room moving & 0.0006 & 0.0107 & 0.0125 & 0.0103 \\
\hline Eyes open, balance pad & 0.0013 & $<0.0001$ & $<0.0001$ & 0.0005 \\
\hline Eyes closed, balance pad & $<0.000$ I & $<0.000$ I & 0.0015 & 0.0007 \\
\hline $\begin{array}{l}\text { Eyes open, balance pad, } \\
\text { room moving }\end{array}$ & $<0.0001$ & $<0.0001$ & $<0.0001$ & $<0.0001$ \\
\hline
\end{tabular}

Abbreviation: VRHMD, virtual-reality head-mounted display.

falls, only variables with a "very high correlation"30 $(r \geq 0.9)$ were analyzed. This also ensured that any differences between the groups were reliable differences. There was a significant difference in anteroposterior velocity between groups (Table 4). While standing with feet together and eyes closed, participants with a risk of falls changed their tilt in the anteroposterior direction at $0.7 \%$ second compared to $0.4 \%$ second for the control group.

\section{Discussion}

As the world's population continues to age, prevention of adverse outcomes due to falls has become an important public health mission. ${ }^{2,3}$ Assessment of balance is an important part of risk stratification of older adults and evaluating the efficacy of fall-prevention and rehabilitation programs. Recent research has shown that exercise with a focus on balance can have a significant impact in fall reduction, and new technologies can facilitate such training. ${ }^{31,32}$ Commercially available low-cost and portable VRHMDs may provide an additional method that can be used to enhance balance assessment. VRHMDs immerse a participant in a different environment by enveloping a user's visual system, providing the potential of a high level of ecological validity. Additionally, the same 3-D positional tracking that is required to immerse an individual allows accurate measurements of movement while simultaneously providing control over a user's visual stimulus. In this study, we evaluated the differences in postural motion between older adults at risk of falls compared to a group of controls. Postural measurements included velocity, amplitude, maximum displacement, and average displacement for both force-plate and VRHMD measurements for anteroposterior and mediolateral directions. Measurements were captured through the VRHMD and force plate simultaneously, with calculations based on tilt 
Table 4 Differences between groups with risk of falls and controls for reliable variables $(r \geq 0.9)$

\begin{tabular}{|c|c|c|c|c|c|c|}
\hline Measurement & Direction & Sensor & Module & Condition & $\begin{array}{l}\text { Correlation } \\
\text { coefficient }\end{array}$ & $P$-value \\
\hline Velocity $(\mathrm{m} / \mathrm{s})^{*}$ & AP & VR & Baseline & Feet together, eyes closed & 0.91 & 0.04 \\
\hline Velocity (m/s) & $M L$ & FP & Balance & Seated & 0.9 & 0.11 \\
\hline Area $\left(m^{2}\right)$ & & FP & Baseline & Feet together, eyes open & 0.97 & 0.64 \\
\hline Mean displacement (m) & $M L$ & VR & Seated & Seated & 0.9 & 0.73 \\
\hline Area $\left(m^{2}\right)$ & & FP & Baseline & Feet together, eyes closed & 0.98 & 0.74 \\
\hline
\end{tabular}

Note: $* p<0.05$.

Abbreviations: AP, anteroposterior; ML, mediolateral; VR, virtual reality (measurement from headset-position system); FP, force plate (measurement from same).

angle for the VRHMD measurements and center of pressure for the force-plate measurements. Additionally, the effect of the assessment protocol on SSQ score was also evaluated through pre- and postassessments.

Comparisons between those at risk of falls and controls were restricted to the variable-module combinations that showed high reliability $(r \geq 0.9)$ between the first and second visits. Measurements taken from the VRHMD indicated that participants with a risk of falls had faster anteroposterior velocity at rest compared to the controls. A limitation of this approach is that findings were not adjusted for multiple comparisons, due to the small numbers used in this pilot study. However, this finding corroborates recent research using a Wii balance board that prospectively assessed falls in community-dwelling older adults, where faster anteroposterior velocity was associated with increased odds of falling. ${ }^{33}$

The use of a VRHMD for balance assessment provides the advantage of added ecological validity through the addition of depth perception and by blocking out all extraneous visual system information. This additional sensory control comes at the cost of added risk of simulator sickness. There was no significant difference in SSQ overall scores, with only a significant difference in nausea-subscale score on the first visit. However, one participant dropped out due to complaints of simulator sickness. Participant debriefing indicated that the main complaint was a difference in room-rotation speed between balance and reaction modules. Mitigating motion sickness is important, as it has previously been shown that older adults may be more susceptible to simulator sickness than younger adults. ${ }^{34}$ One of the key aspects to this study was the absence of rotating the participant's room view along the longitudinal axis, which corresponds to turning one's head from left to right, compared to prior visual-vestibular conflict work..$^{25}$ Avoiding rotation along the longitudinal axis has become standard practice in modern VR development, due to its adverse effects on simulator sickness.
In addition to providing the ability to measure the tilt of a participant, as has been done in accelerometer-based balance studies, ${ }^{29}$ the combined use of a foam balance pad and the VRHMD's ability to induce visual-vestibular conflict allows the assessment of the relative importance of individual sensory systems to postural control. In both the EquiTest sensory organization test and BESS, this is accomplished by comparing sway under different conditions of reduced somatosensory and/or visual information. ${ }^{11}$ This study made use of the integrated 3-D positional system used in modern VRHMDs, which allows measurement of body tilt without requiring additional equipment, and only knowledge of the participant's height is required to compensate for the positional offset of the VRHMD.

Due to the differences between measuring tilt and center of pressure, this study focused on the ability of each approach to differentiate between different conditions used in isolating sensory information. While previous studies have tried to convert tilt measurements into approximate center-of-pressure measurements, the use of the VRHMD measuring point meant that we could not make the same assumptions regarding the position of the center of mass. We treated the measurements from the two approaches as related but distinct, and instead focused on their ability to distinguish between different sensory-deprivation conditions. This approach has been used before in other systems that measure tilt and center of pressure, such as accelerometers. ${ }^{29}$ Comparisons were made evaluating anteroposterior sway, as that was found to be important in both this study and previous studies. ${ }^{33}$ We found that both the VRHMD and the force plate distinguished differences in anteroposterior amplitude compared to the baseline-eyes-open condition. Only with the comparison of eyes open and eyes closed were the measurements unable to detect a difference. This is important, as it indicates that VRHMD measurements provide similar results as force-plate measurements and may be used in assessing the contributions of different sensory systems to the maintenance of balance. 


\section{Limitations and future work}

This study had a small sample size, with a female:male ratio of 10:3. Due to its exploratory nature and small sample size, the study did not use any correction for multiple comparisons, and this increases type I error. We excluded individuals with an MoCA score $\geq 21$, and concerns over major VRHMD intolerance required that participants be comfortable in a stationary VR scene. The MoCA-score limitation may have limited participants, as it would have excluded potential participants who may have both cognitive and balance impairments. Although no potential participants dropped out due to initial static VR intolerance, the significant premeasurementpostmeasurement difference in nausea-subscale score for the first visit and the fact that a participant dropped out due to simulator sickness would suggest that nausea may be an issue with the assessment. Additionally, recent research has shown that rotation speed along the mediolateral axis has a significant effect on simulator sickness, so this could be tweaked in later iterations to find an optimal speed that still induces vection while mitigating simulator sickness. ${ }^{35}$ The number of modules used during testing could be shortened in future to mitigate motion-sickness issues.

\section{Conclusion}

A modern low-cost VRHMD that tracks head movement was valid, reliable, and comparable to a force plate for assessing balance in older adults. A modern head-mounted display that uses VR for visual perturbation may provide an affordable and portable alternative to traditional mechanical perturbation approaches to measuring the different sensory components of balance.

\section{Acknowledgments}

The authors acknowledge Elizabeth Chmelo and Dianne Limbaugh for their help with study-site coordination and participant scheduling. The authors gratefully acknowledge use of the services and facilities of the Wake Forest Center of Translational Science Institute, funded by the National Center for Advancing Translational Sciences (NCATS), National Institutes of Health through grants UL1TR001420 and NIH P30 AG021332.

\section{Disclosure}

The authors report no conflicts of interest in this work.

\section{References}

1. United Nations. World Population Ageing 2015. New York: UN; 2015

2. Sterling DA, O'Connor JA, Bonadies J. Geriatric falls: injury severity is high and disproportionate to mechanism. J Trauma. 2001;50(1): 116-119.
3. Alexander BH, Rivara FP, Wolf ME. The cost and frequency of hospitalization for fall-related injuries in older adults. Am J Public Health. 1992;82(7):1020-1023.

4. Bergen G, Stevens MR, Burns ER. Falls and fall injuries among adults aged $\geq 65$ years: Unites States, 2014. MMWR Morb Mortal Wkly Rep. 2016;65(37):993-998.

5. Sherrington C, Tiedemann A. Physiotherapy in the prevention of falls in older people. J Physiother. 2015;61(2):54-60.

6. Podsiadlo D, Richardson S. The timed "Up \& Go": a test of basic functional mobility for frail elderly persons. J Am Geriatr Soc. 1991;39(2): 142-148.

7. Vellas BJ, Wayne SJ, Romero L, Baumgartner RN, Rubenstein LZ, Garry PJ. One-leg balance is an important predictor of injurious falls in older persons. J Am Geriatr Soc. 1997;45(6):735-738.

8. Söhsten E, Bittar RS, Staab JP. Posturographic profile of patients with persistent postural-perceptual dizziness on the sensory organization test. J Vestib Res. 2016;26(3):319-326.

9. Fitzpatrick R, McCloskey DI. Proprioceptive, visual and vestibular thresholds for the perception of sway during standing in humans. J Physiol. 1994;478(Pt 1):173-186.

10. Nashner LM. A model describing vestibular detection of body sway motion. Acta Otolaryngol. 1971;72(6):429-436.

11. Bell DR, Guskiewicz KM, Clark MA, Padua DA. Systematic review of the balance error scoring system. Sports Health. 2011;3(3): 287-295.

12. Sloane PD, Baloh RW, Honrubia V. The vestibular system in the elderly: clinical implications. Am J Otolaryngol. 1989;10(6):422-429.

13. Mirelman A, Rochester L, Maidan I, et al. Addition of a non-immersive virtual reality component to treadmill training to reduce fall risk in older adults (V-TIME): a randomised controlled trial. Lancet. 2016; 388(10050):1170-1182.

14. Chiarovano E, de Waele C, MacDougall HG, Rogers SJ, Burgess AM, Curthoys IS. Maintaining balance when looking at a virtual reality three-dimensional display of a field of moving dots or at a virtual reality scene. Front Neurol. 2015;6:164.

15. Howcroft J, Lemaire ED, Kofman J, McIlroy WE. Elderly fall risk prediction using static posturography. PloS One. 2017;12(2): e0172398.

16. Menzies RJ, Rogers SJ, Phillips AM, et al. An objective measure for the visual fidelity of virtual reality and the risks of falls in a virtual environment. Virtual Real. 2016;20(3):173-181.

17. Robert MT, Ballaz L, Lemay M. The effect of viewing a virtual environment through a head-mounted display on balance. Gait Posture. 2016;48:261-266.

18. Brigham and Women's Hospital. Strategies to reduce injuries and develop confidence in elders (STRIDE). Available from: https:// clinicaltrials.gov/ct2/show/study/NCT02475850. NLM identifier: NCT02475850. Accessed June 28, 2017.

19. Callahan CM, Unverzagt FW, Hui SL, Perkins AJ, Hendrie HC. Six-item screener to identify cognitive impairment among potential subjects for clinical research. Med Care. 2002;40(9):771-781.

20. Montreal Cognitive Assessment. MoCA Test-English. Version 7.1 2015. Available from: http://www.mocatest.org/wp-content/ uploads/2015/tests-instructions/MoCA-Test-English_7_1.pdf. Accessed September 11, 2015.

21. Guerra RO, Oliveira BS, Alvarado BE, et al. Validity and applicability of a video-based animated tool to assess mobility in elderly Latin American populations. Geriatr Gerontol Int. 2014;14(4):864-873.

22. Bouchard S, Robillard G, Renaud P. Revising the factor structure of the Simulator Sickness Questionnaire. Annu Rev Cyberther Telemed. 2007;5:128-137.

23. Akiduki H, Nishiike S, Watanabe H, Matsuoka K, Kubo T, Takeda N. Visual-vestibular conflict induced by virtual reality in humans. Neurosci Lett. 2003;340(3):197-200.

24. Clinton Industries. Adjustable balance platform. 2015. Available from: http://www.clinton-ind.com/products/physical-therapy-equipment/ exercise-aids/adjustable-balance-platform. Accessed July 27, 2017. 
25. Rosenthal S. Professional Football Career Length and Positions: Potential Risk Factors for Concussive and Subconcussive Exposure Outcomes [master's thesis]. Pennsylvania: Pennsylvania State University; 2014.

26. Moss JD, Muth ER. Characteristics of head-mounted displays and their effects on simulator sickness. Hum Factors. 2011;53(3):308-319.

27. Duarte M, Freitas SM. Revision of posturography based on force plate for balance evaluation. Rev Bras Fisioter. 2010;14(3):183-192.

28. Fernie GR, Gryfe CI, Holliday PJ, Llewellyn A. The relationship of postural sway in standing to the incidence of falls in geriatric subjects. Age Ageing. 1982;11(1):11-16.

29. Mayagoitia RE, Lötters JC, Veltink PH, Hermens H. Standing balance evaluation using a triaxial accelerometer. Gait Posture. 2002;16(1): 55-59.

30. Mukaka MM. A guide to appropriate use of correlation coefficient in medical research. Malawi Med J. 2012;24(3):69-71.

31. Sherrington C, Tiedemann A, Fairhall N, Close JC, Lord SR. Exercise to prevent falls in older adults: an updated meta-analysis and best practice recommendations. N S W Public Health Bull. 2011;22(3-4):78-83.
32. Verrusio W, Gianturco V, Cacciafesta M, Marigliano V, Troisi G, Ripani M. Fall prevention in the young old using an exoskeleton human body posturizer: a randomized controlled trial. Aging Clin Exp Res. 2017;29(2):207-214.

33. Kwok BC, Clark RA, Pua YH. Novel use of the Wii balance board to prospectively predict falls in community-dwelling older adults. Clin Biomech (Bristol, Avon). 2015;30(5):481-484.

34. Kawano N, Iwamoto K, Ebe K, et al. Slower adaptation to driving simulator and simulator sickness in older adults. Aging Clin Exp Res. 2012;24(3):285-289.

35. Dennison MS, D'Zmura M. Cybersickness without the wobble: experimental results speak against postural instability theory. Appl Ergon. 2017;58:215-223
Clinical Interventions in Aging

\section{Publish your work in this journal}

Clinical Interventions in Aging is an international, peer-reviewed journal focusing on evidence-based reports on the value or lack thereof of treatments intended to prevent or delay the onset of maladaptive correlates of aging in human beings. This journal is indexed on PubMed Central, MedLine,

\section{Dovepress}

CAS, Scopus and the Elsevier Bibliographic databases. The manuscript management system is completely online and includes a very quick and fair peer-review system, which is all easy to use. Visit http://www.dovepress. com/testimonials.php to read real quotes from published authors. 